Relations industrielles

Industrial Relations

\title{
L'amélioration de langage de la profession
}

L'expérience d'un comité paritaire et d'une commission

d'apprentissage

\section{Léonce Girard}

Volume 5, numéro 9, juin 1950

URI : https://id.erudit.org/iderudit/1023388ar

DOI : https://doi.org/10.7202/1023388ar

Aller au sommaire du numéro

Éditeur(s)

Département des relations industrielles de l'Université Laval

ISSN

0034-379X (imprimé)

1703-8138 (numérique)

Découvrir la revue

Citer cet article

Girard, L. (1950). L'amélioration de langage de la profession : l'expérience d'un comité paritaire et d'une commission d'apprentissage. Relations industrielles / Industrial Relations, 5(9), 81-83. https://doi.org/10.7202/1023388ar

Tous droits réservés $@$ C Département des relations industrielles de l’Université Laval, 1950
Ce document est protégé par la loi sur le droit d'auteur. L'utilisation des services d'Érudit (y compris la reproduction) est assujettie à sa politique d'utilisation que vous pouvez consulter en ligne.

https://apropos.erudit.org/fr/usagers/politique-dutilisation/ 


\title{
Bulletin des relations industrielles
}

\section{L'AMÉLIORATION DU LANGAGE DE LA PROFESSION}

\author{
L'expérience d'un comité paritaire et d'une commission d'apprentissage
}

\section{LÉONCE GirARD}

Pour s'intéresser aux questions sociales, il n'est pas nécessaire d'en référer à une multitude de principes difficiles et compliqués. Il y a pourtant certaines données simples et fondamentales qu'il ne faut pas oublier. Entre autres, celle-ci: lorsque l'Etat donne aux individus les moyens d'agir, ils règlent eux-mêmes les problèmes de leur profession tout aussi bien, sinon mieux, que les corps publics ne sauraient le faire.

Puisque le Bulletin des relations industrielles m'en fournit l'occasion, je m'efforcerai de vous démontrer la valeur de ce principe en l'appliquant à l'un des plus grands problèmes de la profession, celui d'améliorer le langage technique, et surtout l'expression française propre au métier. Dans l'espace de cinq ans, le comité paritaire et la commission d'apprentissage de la chaussure ont réalisé une réforme de portée vraiment nationale, non seulement en dotant l'industrie d'une terminologie française, mais encore en la faisant accepter graduellement par un nombre considérable d'employeurs et d'employés.

Si un problème semblable se pose dans votre industrie, et si vous croyez que les employés de notre province devraient parler de leur métier avec des termes précis plutôt que dans un jargon demianglais et demi-français, vous serez, sans doute, très intéressés à connaître cette expérience et, peutêtre même, à la réaliser dans votre milieu.

\section{Le problème}

Les membres du comité paritaire, appelés à rédiger le décret, et les représentants de la commission d'apprentissage, chargés d'enseigner le métier, ont vite constaté que nombre de termes utilisés dans l'industrie de la chaussure étaient, non seulement impropres, mais tout simplement affreux. Le même problème se présente, d'ailleurs, dans plusieurs autres industries.

La machinerie, l'outillage, les catalogues nous parviennent, pour la plupart, des Etats-Unis. Les personnes employées dans l'industrie apprennent d'abord les termes anglais et les utilisent en parlant français. Ainsi le patronniste fait son «standard » ou son «spider web»; le tailleur découpe des «vamp», des «back strap», des «quarter», des « overlay»; l'homme de «stock» « channelle» la fausse; le monteur «trimme l'inseam»; le préposé au fonçage «sluggue le top piece», et, au département de la finition, on fait le «burnishage ». La plus élémentaire fierté du métier exigeait que la profession améliorât son langage.

\section{Le comité}

Un comité a été chargé de trouver et de compiler les termes du métier: termes anglais officiels et, en regard, les termes français. Le comité a travaillé dur. La tâche n'était pas facile. Les termes français étaient si différents des expressions anglaises, qu'en lisant les livres français on ne les comprenait pas.

Enfin, après avoir comparé les machines, les dessins, les photos, les volumes, les documents, le comité a rédigé, tout d'abord, une liste qui a été approuvée par l'assemblée, puis une brochure miméographiée de 36 pages avec illustrations.

Voici, à titre d'exemple, quelques expressions anglaises et leurs correspondants français:

$\begin{array}{lll}\text { rough rounding } & - & \text { graver sur forme } \\ \text { channelling insole } & - & \text { graver la première } \\ \text { laying sole } & \text { ficher la semelle } \\ \text { slugging heel } & - & \text { bonbouter le talon } \\ \text { trimming edge } & - & \text { fraiser la lisse } \\ \text { fixing wedge heel } & - & \text { poser le talon cambrure } \\ \text { cutting back strap } & - & \text { tailler la baguette } \\ \text { burnishing heel } & - & \text { lustrer le talon } \\ \text { skiving vamp } & - & \text { parer lempeigne }\end{array}$

Les noms des membres de ce comité ne passeront probablement pas à l'histoire; il n'en reste pas moins qu'ils ont rendu un immense service à la cause de la langue française dans la province.

\section{Diffusion}

Connaître les termes, c'était le premier pas. Mais comment les faire adopter par la masse ? Un comité paritaire et une commission d'apprentis- 
sage ont toujours des moyens à leur disposition, précisément parce qu'ils ont le grand avantage d'avoir des contacts immédiats et journaliers avec les patrons et ouvriers de l'industrie.

Tous ces contacts ont été mis à contribution. D'abord, les termes ont été insérés dans le décret; chacune des opérations a été décrite avec les termes choisis, anglais et français. Puis, les inspecteurs du comité paritaire, qui entrevoient chaque salarié individuellement, au moins tous les trois mois, ont enseigné, à chacun des employés de langue française, le terme français de son opération. Le «trimmer» de lisses a su que son opération s'appelait «fraiser la lisse»; le «rounder» a appris que son travail s appelait «graver sur forme »; le patronniste a fait un gabarit au lieu d'un «standard » et le tailleur a découpé des empeignes, des quartiers, des baguettes et des appliqués.

Plusieurs manufacturiers ont demandé les termes français et ont fait imprimer leurs listes de prix, en anglais et en français, de sorte que maintenant l'employé à la pièce peut lire les deux termes chaque fois qu'il détache un coupon de paye.

Pour sa part, la commission d'apprentissage utilise les termes français dans la rédaction de ses cours et dans l'analyse des opérations. Les professeurs de l'école ont instruction d'utiliser les termes français afin que les travailleurs en chaussures, finissants de l'école, soient familiers avec ces termes.

La commission d'apprentissage est allée plus loin. Elle a donné, dans les écoles de chaussure, dans les usines, dans les syndicats ouvriers, à travers la province, des cours abrégés à tout groupe de 10 à 15 employés qui en faisaient la demande; l'instructeur a expliqué, avec parties de chaussures en mains, chacun des morceaux et chacune des opérations en indiquant l'expression française exacte.

Et nous avons reçu des secours inattendus. Des contremaîtres, intéressés à la cause, ont inscrit, chaque jour, dans leurs départements, sur tableau noir, les noms français de quelques opérations pour s'assurer que chaque employé les apprendrait et les utiliserait.

Mentionnons encore que le «Service de renseignements » a adressé à tous les manufacturiers et salariés de l'industrie de la chaussure des listes complètes des principaux termes français et anglais de chaque département, avec illustrations. Les termes français ou anglais, selon le cas, sont employés dans chaque bulletin.

Voici, en résumé, les moyens utilisés par le comité paritaire et la commission d'apprentissage pour vulgariser les termes français, et même les termes anglais exacts, dans l'industrie:
1. Le comité paritaire:

- revision et correction du décret;

- entrevues des inspecteurs avec chaque employé;

- revision des listes de prix ou coupons de paye;

- propagande à domicile par le «Service de renseignements ».

\section{La commission d'apprentissage:}

- rédaction des cours en français;

- enseignement des termes par les professeurs à l'école;

- cours abrégés dans les usines et les organisations syndicales;

- collaboration des contremaîtres, dirigeants de personnel et chefs ouvriers.

Entre nous, y a-t-il un pouvoir public qui pourrait utiliser autant de moyens à la fois pour arriver à ses fins? Pour cela, il faut connaitre l'industrie dans ses détails et chacune des personnes qui la composent. Solutionner les problèmes de la profession appartient, en premier lieu, aux patrons et ouvriers de l'industrie; c'est le rôle de l'Etat, non pas de se substituer aux intéressés, mais de seconder leurs efforts, comme il le fait, d'ailleurs, par la Loi de la convention collective et par la Loi de l'aide à l'apprentissage.

\section{Résultats obtenus}

Tout n'est pas encore parfait, loin de là. Mais la marche continue et le langage professionnel s'améliore constamment. Nous en avons des preuves tous les jours. Nous recevons, par exemple, des lettres de manufacturiers ou de leurs représentants, d'organisations ouvrières ou de contremaîtres, qui contiennent exclusivement des termes français au lieu des expressions courantes d'autrefois. En plus, des bureaux de traducteurs nous demandent des termes français. Des tanneurs, des industriels, des fournisseurs, non seulement de la province de Québec, mais de l'Ontario et des EtatsUnis, s'assurent que leurs annonces portent bien les termes français du métier à côté de l'expression anglaise exacte.

Tout cela s'est fait dans un espace de temps relativement court, ce qui nous laisse espérer que, dans quelques années, tous les employeurs et employés de la chaussure utiliseront les termes techniques français lorsqu'ils parleront français. 
Les comités paritaires et les commissions d'apprentissage, tout comme les gouvernements, les pouvoirs publics, les professions libérales et autres, ne sont pas parfaits. Mais si nous considérons le travail qu'ils ont accompli en si peu de temps, et sans causes sérieuses de critique, il n'y a pas de doute qu'ils peuvent soutenir la comparaison avec les institutions les plus efficaces et les plus méritantes.
Améliorer le langage de la profession n'est qu une bien faible partie de la tâche qui incombe aux comités paritaires et aux commissions d'apprentissage; mais, à mon point de vue, ce travail est si important, si nécessaire et si urgent, que si les comités et les commissions n'avaient fait que cela, ils auraient déjà prouvé leur raison d'être et bien davantage.

\title{
NATURE JURIDIQUE ET DÉVELOPPEMENT HISTORIQUE DU DROIT DU TRAVAIL
}

\author{
René H. Mankiewicz
}

\section{Définition}

Le droit du travail peut être défini comme étant «l'ensemble des règles qui, dérogeant au droit commun ou le complétant, régissent les droits et les devoirs des personnes fournissant ou acceptant un travail dépendant». Peu importe qu'il y ait un contrat de travail valide: les droits et obligations résultent du fait de la prestation ou de l'acceptation d'un travail fourni sous la direction d'autrui. Ainsi il a été admis par certains tribunaux que le salaire prévu par la convention collective ou par tout autre règlement est dû, même en cas de nullité du contrat de travail, par exemple, lorsqu'un mineur a loué ses services sans avoir eu la capacité juridique de le faire. De même la nullité du contrat ne s'oppose pas, dans la plupart des législations, à l'exercice des droits que le travailleur peut avoir contre une assurance sociale, etc.

C'est par la dépendance où il se trouve à l'égard de son employeur que le travailleur au sens du droit du travail se distingue de tout autre individu fournissant une oeuvre à autrui. Cette dépendance se comprend dans un sens purement matériel: elle signifie une subordination réelle du travailleur à l'entrepreneur, et trouve son expression la plus nette dans le «droit de direction» qui permet à celui-ci de prescrire au travailleur la nature de son ouvrage, les conditions, les heures, etc. de son exécution.

Il y a cependant des personnes qui, sans être soumises à une subordination et tout en restant maîtresses de leur activité et de son aménagement matériel, se trouvent néanmoins dans une très forte dépendance économique vis-à-vis de leur commettant: travailleurs à domicile, coiffeurs locataires de leur fauteuil, chauffeurs de taxis, etc...
Le droit du travail les «assimile» aux travailleurs dépendants à raison précisément de ce manque d'indépendance et de liberté de mouvement économique.

Il y a, d'autre part, des personnes au service d'autrui qui gardent dans une large mesure leur autonomie et leur liberté d'action, restant en fait maîtresses de leur activité et de l'emploi de leur temps. C'est plus particulièrement le cas des administrateurs et directeurs de sociétés. Ils sont subordonnés à la société, personne morale, représentée par l'assemblée générale ou tout autre organe qualifié. Mais ces organes ne peuvent les diriger et surveiller à tout moment. Aussi les directeurs, administrateurs et autres représentants légaux de personnes juridiques jouent-ils normalement le rôle, non pas de travailleurs, mais d'employeurs, exerçant à la place de la personne morale les droits et les devoirs imposés par le droit du travail à celui qui accepte un travail dépendant. Cette dernière règle n'est pas toutefois sans exceptions: leur nombre et leur étendue varient avec les rapports réels qui existent entre le directeur et la société, ainsi que selon les différentes législations nationales. Sur toutes ces questions, on trouve des décisions judiciaires très instructives dans les douze volumes du Recueil international de jurisprudence de travail, édité par le B.I.T. De 1925 à 1938/39 ce Recueil analysait les arrêts les plus importants rendus en matière de droit du travail par les cours de justice françaises, allemandes, américaines, anglaises et italiennes. On ne peut que regretter que le B.I.T. n'ait pu reprendre cette publication après la guerre.

Les normes du droit du travail, établies par la loi, les ordonnances administratives, les arrêts 